\title{
Predictive parameters in hypofractionated whole- breast 3D conformal radiotherapy according to the Ontario Canadian trial
}

This article was published in the following Dove Press journal:

OncoTargets and Therapy

24 March 2017

Number of times this article has been viewed

\author{
Grazia Lazzari' \\ Angela Terlizzi \\ Giuseppina Della Vittoria \\ Scarpati ${ }^{3}$ \\ Francesco Perri ${ }^{3}$ \\ Vincenzo De Chiara ${ }^{4}$ \\ Barbara Turi \\ Giovanni Silvano' \\ 'Radiation Oncology Unit, ${ }^{2}$ Physics \\ Department, ${ }^{3}$ Medical Oncology Unit, \\ Saint Giuseppe Moscati Hospital, \\ ${ }^{4}$ Radiation Therapy Unit, Saints \\ Giovanni Di Dio and Ruggi di Aragona, \\ University of Salerno, Taranto, Italy
}

Correspondence: Grazia Lazzari

Radiation Oncology Unit, Saint Giuseppe Moscati Hospital, Road to Martina Franca, 74100 - Taranto, Italy

Tel+390994585721

Email lazzarigrazia@gmail.com
Aim: To evaluate the possible role of dosimetric parameters according Normal Tissue Complication Probability (NTCP) model as predictive of late toxicity and cosmesis in hypofractionated whole-breast three-dimensional conformal radiotherapy.

Patients and methods: A retrospective analysis on 215 consecutive early breast cancer patients treated with breast conserving surgery and adjuvant hypofractionated whole-breast radiotherapy (according the Ontario Canadian trial), with a 6 years median follow-up was conducted. To assess the impact of $10 \%-20 \%$ dose hotspots on different percent values of planning target volume (PTV) of the breast, we retrospectively employed the NTCP model of Lyman. PTV breast (PTVbr), V110 were identified. For statistical analysis the $\chi^{2}$ and paired $t$-test were used to find a correlation between late skin and subcutaneous toxicity and cosmetic outcome with dosimetrical parameters Multivariate analysis was performed with the aim to assess independently the impact of dosimetric and clinical parameters on late toxicity and cosmesis using Pearson's covariance.

Results: Late skin toxicity was recorded in 47/215 (22\%); and G3 toxicity occurred in 11 patients $(5 \%)$. Cosmesis with excellent-good score was found in 172 patients $(80 \%)$ while fair-poor score was found in 43 patients (20\%). In univariate $\chi^{2}$ analysis the V110 $>10 \%$ of the PTV breast significantly correlated with higher toxicity $(P<0.005$, OR 9.60 [CI 3.89-23.72]). Cosmesis related to $\mathrm{V} 110>10 \%$ and PTV breast volume over $1,300 \mathrm{cc}$ was significant at multivariate analysis $(P<0.005$, OR 6.07 [CI 2.36-15.59]).

Conclusion: To safely use one of the most important whole-breast hypofractionated radiotherapy schedules, we found some predictive paramaters on the basis of NTCP model by Lyman. These parameters may be useful in selection of elegible patients.

Keywords: NTCP model, Ontario Canadian Trial, hypofractionated radiotherapy, whole-breast, cosmesis, toxicity, PTV breast, V110, early breast cancer

\section{Introduction}

In the last decade, many randomized trials have investigated the safety and efficacy of hypofractionation for adjuvant whole-breast irradiation in light of the greater treatment time convenience ${ }^{1}$ and the unchanged results in local control in comparison with the conventional fractionation. ${ }^{2}$ A systematic review and meta-analysis comparing hypofractionated radiotherapy with conventional radiotherapy has confirmed no significant difference between the two arms in locoregional recurrence, overall survival, late normal tissue toxicity, and cosmetic outcome. ${ }^{3}$ Furthermore, another review and meta-analysis on randomized controlled trials recommends moderately hypofractionated radiotherapy as a standard adjuvant treatment in the majority of patients with breast cancer. ${ }^{4}$ The 
well-known moderately and gentler randomized hypofractionated radiotherapy schedule proposed by the Ontario Canadian Trial $^{5}$ has clearly shown at 5-10 years a good local control (locoregional recurrence $3.0 \%$ vs $2.8 \%$ ) and a good cosmetic outcome (breast appearance $77 \%$ vs $77 \%$ ) of the experimental hypofractionated arm, ${ }^{6}$ leading to its approval in the clinical practice. Hypofractionated radiotherapy is supported by radiobiological principles according to a linear-quadratic model for different $\alpha / \beta$ ratios of breast cancers, normal breast tissue, and surrounding organ at risk. ${ }^{7}$ Because it is delivered with larger daily fractions, there are clinical and dosimetric concerns about the effects of hotspots on fraction sizes, dose heterogeneity, and breast volume sizes as questioned by Yarnold et $\mathrm{al}^{8}$ in a critical review. The American Society for Radiation Oncology (ASTRO) guidelines in 2011 have also aimed to answer these questions with meticulous care to optimize dose homogeneity within the target volume using three-dimensional (3D) conformal hypofractionated radiotherapy. ${ }^{9}$ In fact, the Ontario Canadian Trial provides data concerning a two-dimensional (2D) radiotherapy technique used upon a well selected population in which few patients have been treated with chemotherapy and large-breasted women have been excluded to prevent dose target heterogeneity, ${ }^{6}$ so no more information regarding the impact of these factors on toxicity and cosmetic outcome is available to be applied on a hypofractionated whole-breast 3D conformal radiotherapy (HF-WB 3D-CRT). This retrospective study aimed to analyze the correlation of dosimetric and clinical parameters with cosmesis and late toxicity in patients treated with HF-WB 3D-CRT according to the randomized Ontario Canadian Trial.

\section{Materials and methods}

\section{Characteristics of patients and data collection}

From 2004 to 2011, 215 consecutive patients were recruited for HF-WB 3D-CRT according to these primary inclusion criteria: 1) postmenopausal age $>60$ years; 2 ) pathological stage pT1-T2 pN0 M0 invasive breast cancer according to the American Joint Committee on Cancer and Union for International Cancer Control; and 3) quadrantectomy and axillary clearance or sentinel node sampling. The use of adjuvant chemotherapy or hormone therapy was permitted according to the recent clinical guidelines in all the patients as shown in Table 1. Unlike the Ontario Canadian Trial, the breast diameter (BD) as the breast width exceeding $25 \mathrm{~cm}$ at the posterior border of the medial and lateral tangential beams was not considered as an exclusion criterion. A written informed consent to the treatment and analysis of the data was provided by all the patients for this study. The ethical
Table I Characteristics of patients and clinical parameters

\begin{tabular}{ll}
\hline Patients & 215 \\
Mean age (years) & $68(60-75)$ \\
Stage pTI pN0/pT2 pN0 & $55 / 160$ \\
Chemotherapy/hormonal & $80 / 135$ \\
Antracycline-containing scheme & 44 \\
Cyclophosfamide-methotrexate-5- & 36 \\
fluorouracil & \\
Good surgery score & 170 \\
Fair surgery score & 45 \\
Mean breast volume & $1,250 \mathrm{cc}(560-1,500)$ \\
Breast separation diameter $<25 \mathrm{~cm}$ & 135 \\
Breast separation diameter $>25 \mathrm{~cm}$ & 80 \\
\hline
\end{tabular}

approval was obtained from the Perrino Hospital of Brindisi Ethic Committee for this study.

\section{Radiation treatment}

Hypofractionation was delivered to all the 215 eligible patients. The patients were treated with 3D-CRT planned with Masterplan Treatment Planning ${ }^{\circledR}$ (Nucletron version 1, Elekta, Crawley, UK). The planning computed tomography (CT) scan had a slice thickness of $5 \mathrm{~mm}$ on chest starting from cricoid to diaphragm; all the patients were treated in supine position on an immobilization device with shoulder inclination and both arms above the head. The clinical target volume (CTV) consisting of the whole remaining breast volume was defined as the palpable breast included in a tangential field, excluding the deep structures. The planning target volume (PTV) of breast consisted of CTV expanded by a margin of $1 \mathrm{~cm}$ below and over the palpable breast for breathing motion and treatment setup and $0.5 \mathrm{~cm}$ under the skin line and over the rib as seen on the CT images. The whole breast was treated using -2-4 opposed tangential multileaf collimator customized fields-in-fields technique and 6-10 MV photon beams, depending on the breast size and PTV breast (PTVbr) coverage. The prescribed dose (PD) was $42.56 \mathrm{~Gy}$ in 16 fractions (266 cGy/fr, 5 fr/week), and no boost was added. The dose was prescribed to the International Commission on Radiation Units \& Measurements (ICRU) reference point according to the ICRU-PTV constraints. For this analysis, the following dosimetric parameters were identified: PTVbr as the residual breast with margin expansions as before, V110 as the percentage of the PTVbr receiving $>10 \%$ of PD, PTV (cc) including the mean-cc breast volume calculated in the dose-volume histogram (DVH) table, and $\mathrm{BD}$ as the breast width at the posterior border of the medial and lateral tangential beams measured on the isocenter CT slice. For each patient, DVHs for PTVbr and organ at risks were obtained. All patients were treated with an Elekta Precise ${ }^{\circledR}$ accelerator (Elekta). 


\section{The NTCP (normal tissue complication probability) predictive model}

To assess the impact of $10 \%-20 \%$ of the PD hotspots on different percent values of the PTVbr and the possible occurrence of double-trouble and triple-trouble effects, the NTCP model by Lyman dose-volume plot for late normal tissue was retrospectively employed. For the biologic equivalent dose, as used in the Ontario Canadian Trial, the $\alpha / \beta=3.4$ Gy was considered for late change of breast appearance. By this model calculating the equivalent dose at 2 Gy fraction for alpha/beta ratio 3.4 (EQD2 3.4 ) for hotspots of $10 \%-20 \%$ of the PD, a dosimetric parameter was identified: V110 >10\% to indicate hotspot $>110 \%$ of PD to more than $10 \%$ of PTVbr as a predictive parameter of late toxicity. ${ }^{10}$

\section{Follow-up, cosmesis, and late toxicity assessment}

The follow-up consisted of clinical evaluation and pictures taken every 3 months after radiotherapy during the first year, every 6 months for the following 3 years, and then yearly. Cosmesis and late toxicity of the treated breast were compared with those the untreated breast. In conformity with the Ontario Canadian Trial, cosmesis was graded according to the European Organisation for Research and Treatment of Cancer (EORTC) cosmetic rating system, ${ }^{11}$ and late skin toxicity was evaluated by a physician and a nurse according to the Radiation Therapy Oncology Group/EORTC scale (Version 2). ${ }^{12}$ At the baseline, for each patient, a surgical cosmesis score was obtained by applying the cosmetic scoring criteria, good or poor, by Taylor in $1995 .{ }^{13}$

\section{Statistical analysis}

The $\chi^{2}$ and paired $t$-tests were used to evaluate the correlation between late skin toxicity and cosmetic outcome with dosimetric parameters so identified by PTVbr, V110, BD more or less $25 \mathrm{~cm}$ on the isocenter CT slice, and clinical information such as chemotherapy and surgery score. The multivariate analysis was performed with the aim to independently predict the impact of dosimetric and clinical parameters on late toxicity and cosmesis using Pearson's covariance correlation test. The statistical significance was assumed at $P<0.05$; data were processed using the SPSS Version 2.1 (SPSS Inc., Chicago, IL, USA) by normal license.

\section{Results}

For late toxicity and cosmesis, 6-year median follow-up data from all the 215 patients were collected; patient characteristics and details are shown in Table 1. Skin late toxicity was recorded in 47 patients (22\%); grade 3
(G3) toxicity occurred in 11 patients (5\%). Cosmesis with excellent-good score resulted in 172 patients (80\%) while fair-poor score in 43 patients $(20 \%)$ as in Table 2 . To identify the role of dosimetric parameters, BD was calculated retrospectively, and the plan was analyzed with respective DVH among patients who developed toxicity and who did not calculate the effect of V110 with the NTCP model by Lyman dose-volume plot for late normal tissue.

Considering for $266 \mathrm{cGy} \times 16$ fraction, the EQD2 ${ }_{3.4}$ is 47.8 Gy and biologically effective dose (BED) is $75.9 \mathrm{~Gy}$; with $10 \%$ hotspots, the result is $290 \mathrm{cGy} \times 16$ fraction with EQD $2_{3.4}$ 54.2 Gy (see Equation 1) and 86 Gy BED (see Equation 2); with $20 \%$ hotspots, the result is 320 cGy $\times 16$ fraction with EQD2 ${ }_{3.4} 62.6$ Gy (see Equation 3) and 99.4 Gy BED (see Equation 4).

$$
\begin{gathered}
\operatorname{EQD}^{2}=n \cdot\left(d+h_{10 \%}\right) \cdot \frac{\left(d+h_{10 \%}\right)+(\alpha / \beta)_{\text {normal breast }}}{2+(\alpha / \beta)_{\text {normal breast }}} \\
\mathrm{BED}=n \cdot\left(d+h_{10 \%}\right) \cdot\left[1+\frac{\left(d+h_{10 \%}\right)}{(\alpha / \beta)_{\text {normal breast }}}\right] \\
\mathrm{EQD} 2=n \cdot\left(d+h_{20 \%}\right) \cdot \frac{\left(d+h_{20 \%}\right)+(\alpha / \beta)_{\text {normal breast }}}{2+(\alpha / \beta)_{\text {normal breast }}} \\
\mathrm{BED}=n \cdot\left(d+h_{20 \%}\right) \cdot\left[1+\frac{\left(d+h_{20 \%}\right)}{(\alpha / \beta)_{\text {normal breast }}}\right]
\end{gathered}
$$

On the plot for NTCP and percentage of PTVbr for EQD2 ${ }_{3.4}$ in the cases of hotspots of $10 \%$ and $20 \%$, respectively, a high value of NTCP was obtained for hotspot of $10 \%$ given to more than $10 \%$ of the PTVbr (Figure 1). For patients who developed toxicity or worse cosmetic hotspots $>110 \%$ $(110 \%-113 \%)$ to a mean $13 \%(10 \%-15 \%)$ of PTVbr were observed.

\section{Univariate analysis}

Late toxicity correlated with V110 $>10 \%$ of the PTVbr significantly $(P<0.005$, odds ratio (OR) 9.60 [confidence interval (CI) 3.89-23.72]); for PTVbr, there was a statistical relation of PTVbr $>1,300 \mathrm{cc}$ and toxicity $(P<0.003$, OR 16.67

Table 2 Results of late toxicity and cosmesis at mean 6-year follow-up

\begin{tabular}{ll}
\hline Toxicity GI/G2 & 47 patients (22\%) \\
Toxicity G3 (skin and subcutaneous) & I I patients (5\%) \\
Cosmesis (excellent/good) & I 72 patients (80\%) \\
Cosmesis (fair/poor) & 43 patients (20\%) \\
\hline
\end{tabular}

Abbreviation: G, grade. 


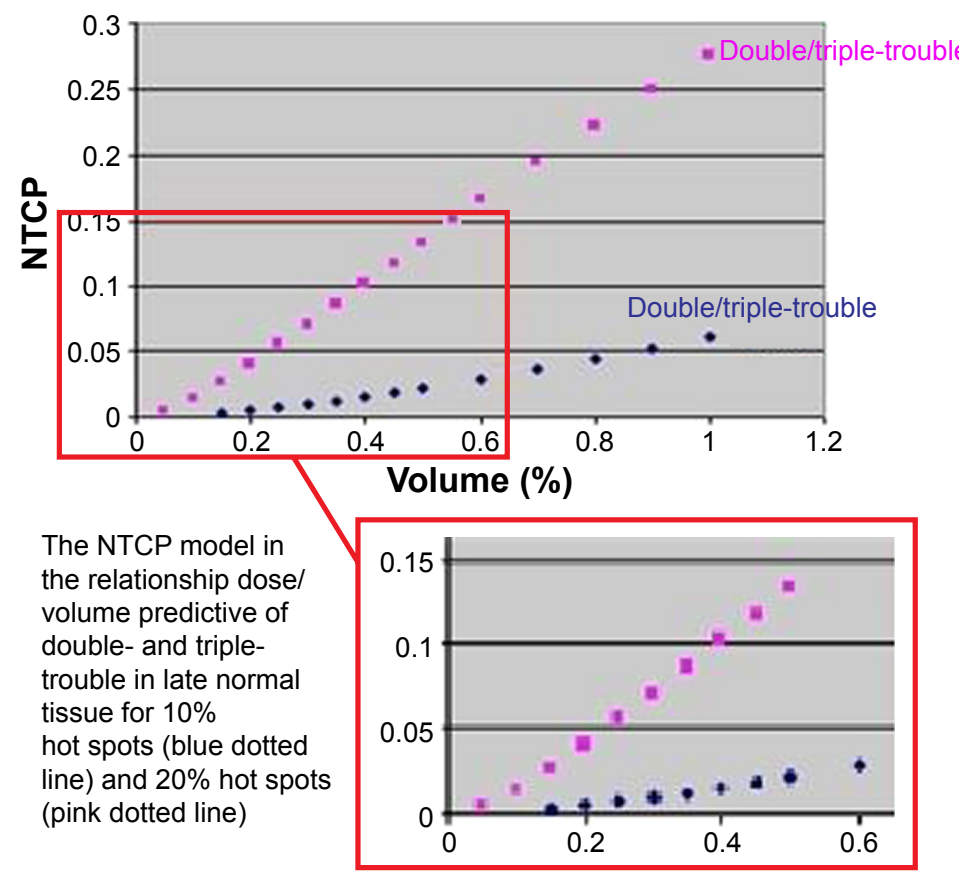

\section{Lyman's model for NTCP*}

$$
\begin{aligned}
& \mathrm{NTCP}=\frac{1}{\sqrt{2 \pi}} \int_{-\infty}^{r} \exp \left(\frac{-x^{2}}{2}\right) d x=\frac{1}{2}\left[1+\operatorname{erf}\left(\frac{t}{\sqrt{2}}\right)\right] \\
& \mathrm{t}=\frac{D-T D_{20}(v)}{m^{*} T D_{50}(v)} \quad T D_{50}(v)=T D_{50}(1) x_{v}^{-z} \\
& T D_{50}(1)=70 \text { Gy } \\
& \mathrm{n}=0.10 \\
& \mathrm{n}=0.12 \\
& \alpha / \beta=3.4 \text { for normal breast }
\end{aligned}
$$

\begin{tabular}{|llll|}
\hline & Gy/fz & EQD2 (Gy) & BED (Gy) \\
\hline $\begin{array}{l}\text { Standard } \\
\text { fraction }\end{array}$ & $2.66 \mathrm{~Gy} /$ & 47.8 & 75.9 \\
Hotspots & $2.9 \mathrm{dGy} /$ & 54.2 & 86.0 \\
$>10 \%$ of PD & $16 \mathrm{fz}$ & & \\
Hotspots & $3.2 \mathrm{dGy} /$ & 62.6 & 99.4 \\
$>20 \%$ of PD & $16 \mathrm{fz}$ & & \\
\hline
\end{tabular}

Figure I The NTCP model for late tissue predicts the double-trouble (according to higher fraction size) and triple-trouble (according to high total dose) effects for hotspots $>10 \%$ of PD (blue dotted line) and for hotspots $>20 \%$ of PD (pink dotted line). ${ }^{*}$ The formula refers to Lyman's model ${ }^{18}$ but the original data from this study has been applied.

Abbreviations: NTCP, normal tissue complication probability; PD, prescribed dose; PTV, planning target volume; BED, biologically effective dose.

[CI 3.06-90.8]) as in Table 3. Cosmesis significantly correlated with V110 $>10 \%$ and PTVbr over 1,300 $\mathrm{cc}(P<0.005$, OR 6.07 [CI 2.36-15.59]). Surgery (good vs poor) significantly correlated with cosmesis and toxicity $(P<0.005, \mathrm{OR}$ 9.75 [CI 4.39-27.6]). Regarding BD, no statistical correlation was found for toxicity with V110 ( $P=0.51$, OR 0.67 [CI 0.20-2.26]) and PTVbr $(P=0.7)$. Chemotherapy did not significantly impact on PTVbr and V110 ( $P=0.53$, OR 1.47 [CI 0.43-4.99]). The univariate analysis with $t$-test also confirmed the results of the $\chi^{2}$ test as shown in Table 4 .

\section{Multivariate analysis}

Pearson's covariance correlation test confirmed the significant relation of toxicity and cosmesis with PTVbr,

Table 3 Univariate analysis $\left(\chi^{2}\right)$ for relation of late toxicity and cosmesis

\begin{tabular}{lllll}
\hline Parameters & $\begin{array}{l}\text { Tox } \\
\text { G I/G2 }\end{array}$ & $\begin{array}{l}\text { Tox } \\
\text { G3 }\end{array}$ & $\begin{array}{l}\text { Cosmesis } \\
\text { (Exc/good) }\end{array}$ & $\begin{array}{l}\text { Cosmesis } \\
\text { (F/P) }\end{array}$ \\
\hline PTV $<1,300 \mathrm{cc}$ & $P=0.2$ & $P=0.3$ & $P=0.03$ & $P=0.3$ \\
PTV $>1,300 \mathrm{cc}$ & $P=0.16$ & $P=0.003$ & $P=0.2$ & $P=0.005$ \\
VII0 $<10 \%$ & $P=0.6$ & $P=0.5$ & $P=0.004$ & $P=0.5$ \\
VII0 $>10 \%$ & $P=0.5$ & $P<0.005$ & $P=0.1$ & $P=0.005$ \\
\hline
\end{tabular}

Abbreviations: Exc, excellent cosmesis; $F$, fair cosmesis; G, grade; $P$, poor cosmesis; PTV, planning target volume; Tox, late toxicity; VIIO, volume of the PTV breast taking $110 \%$ of the prescribed dose.
V110, and surgery. Moreover, a weak correlation was found between diameter and cosmesis as shown in Table 5

\begin{tabular}{|c|c|c|}
\hline Parameters & Correlation & $P$-value \\
\hline VIIO and toxicity & 0.403 & $<0.001$ \\
\hline PTV and toxicity & 0.955 & $<0.001$ \\
\hline CT and toxicity & -0.085 & 0.021 \\
\hline $\mathrm{BD}$ and toxicity & 0.110 & 0.11 \\
\hline Surgery and toxicity & 0.455 & $<0.001$ \\
\hline Cosmesis and PTV & 0.485 & $<0.001$ \\
\hline Cosmesis and VIIO & 0.938 & $<0.001$ \\
\hline Cosmesis and $\mathrm{CT}$ & 0.022 & 0.750 \\
\hline Cosmesis and $\mathrm{BD}$ & 0.285 & $<0.00$ I \\
\hline Cosmesis and surgery & 0.938 & $<0.001$ \\
\hline \multicolumn{3}{|c|}{ 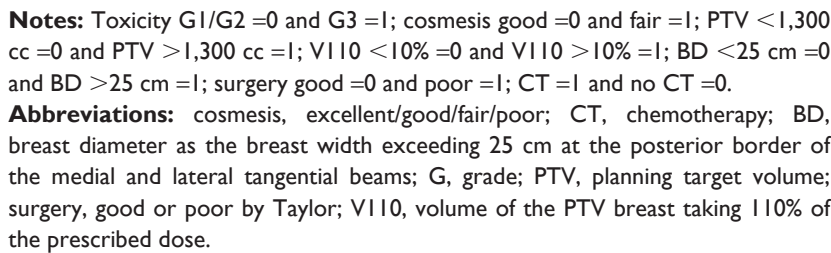 } \\
\hline
\end{tabular}
and Figure 2.

\section{Discussion}

The results of randomized trials comparing the conventional and modestly hypofractionated whole-breast radiotherapy

Table 4 Univariate analysis (paired $t$-test) 
Table 5 Pearson's covariance for multivariate analysis

\begin{tabular}{|c|c|c|c|c|c|c|c|}
\hline & Toxicity & PTV & VIIO & BD & Cosmesis & Surgery & CT \\
\hline \multicolumn{8}{|l|}{ PTV } \\
\hline$R$ & $0.955 * *$ & 1 & $0.430 * *$ & 0.127 & $0.485^{* *}$ & $0.485 * *$ & -0.073 \\
\hline$P$ & $<0.001$ & - & 0.00 & 0.067 & $<0.01$ & 0.000 & 0.289 \\
\hline \multicolumn{8}{|c|}{ VIIO } \\
\hline$R$ & $0.403 * *$ & $0.430 * *$ & I & $0.285^{* *}$ & $0.938 * *$ & $0.969 * *$ & 0.022 \\
\hline$P$ & $<0.001$ & 0.000 & - & 0.000 & $<0.01$ & 0.000 & 0.750 \\
\hline \multicolumn{8}{|l|}{ BD } \\
\hline$R$ & 0.110 & 0.127 & $0.285^{* *}$ & 1 & $0.285^{* *}$ & $0.256 * *$ & -0.019 \\
\hline$P$ & 0.111 & 0.067 & 0.000 & - & $<0.01$ & 0.000 & 0.788 \\
\hline \multicolumn{8}{|c|}{ Surgery } \\
\hline$R$ & $0.455 * *$ & $0.485 * *$ & $0.969 * *$ & 0.256 ** & $0.938 * *$ & 1 & 0.048 \\
\hline$P$ & $<0.001$ & 0.000 & 0.000 & 0.000 & $<0.01$ & - & 0.490 \\
\hline \multicolumn{8}{|l|}{ CT } \\
\hline$R$ & -0.085 & -0.073 & 0.022 & -0.019 & 0.022 & 0.048 & I \\
\hline$P$ & 0.221 & 0.289 & 0.750 & 0.788 & 0.750 & 0.490 & - \\
\hline
\end{tabular}

Notes: Toxicity GI/G2 $=0$ and G3 $=\mathrm{I}$; cosmesis good $=0$ and fair $=\mathrm{I} ; \mathrm{PTV}<\mathrm{I}, 300 \mathrm{cc}=0$ and PTV $>I, 300 \mathrm{cc}=\mathrm{I} ; \mathrm{VII}=10 \%=0$ and VII0 $>10 \%=\mathrm{I} ; \mathrm{BD}<25 \mathrm{~cm}=0 \mathrm{BD}>25$ $\mathrm{cm}=\mathrm{I}$; surgery good $=0$ and poor $=\mathrm{I} ; \mathrm{CT}=\mathrm{I}$ and no $\mathrm{CT}=0$ ). ${ }^{* *} \mathrm{~A}$ very high value of Pearson's correlation number confirmed in the double tail test.

Abbreviations: BD, breast diameter as the breast width exceeding $25 \mathrm{~cm}$ at the posterior border of the medial and lateral tangential beams; cosmesis, excellent/good/ fair/poor; CT, chemotherapy; G, grade; PTV, planning target volume; surgery, good or poor by Taylor; VI I0, volume of the PTV breast taking I I0\% of the prescribed dose; $P$, statistical significance; $R$, correlation number.

schedules have not shown differences in treatment safety, effectiveness, and cosmetic outcomes at a median follow-up of 5-10 years. Hypofractionation was found to be more convenient for patients and less expensive for physicians as emphasized in a recent review. ${ }^{14}$ Long-term results of the Ontario Canadian Trial have clearly demonstrated that the hypofractionated $266 \mathrm{cGy} / \mathrm{fr} \times 16$ schedule is not inferior to standard fractionation in terms of efficacy and safety. On the basis of these findings, data of the Ontario Canadian Trial with a 5-year follow-up reported in the experimental arm that $79 \%$ of the patients showed an excellent-good cosmetic outcome and a value of G2-G3 skin toxicity $<4 \% .{ }^{5}$ Furthermore results with a median follow-up of 12 years confirmed no significant difference between hypofractionation and standard fractionation in terms of local control, cosmetics, and late toxicity so that this schedule has been defined gentler

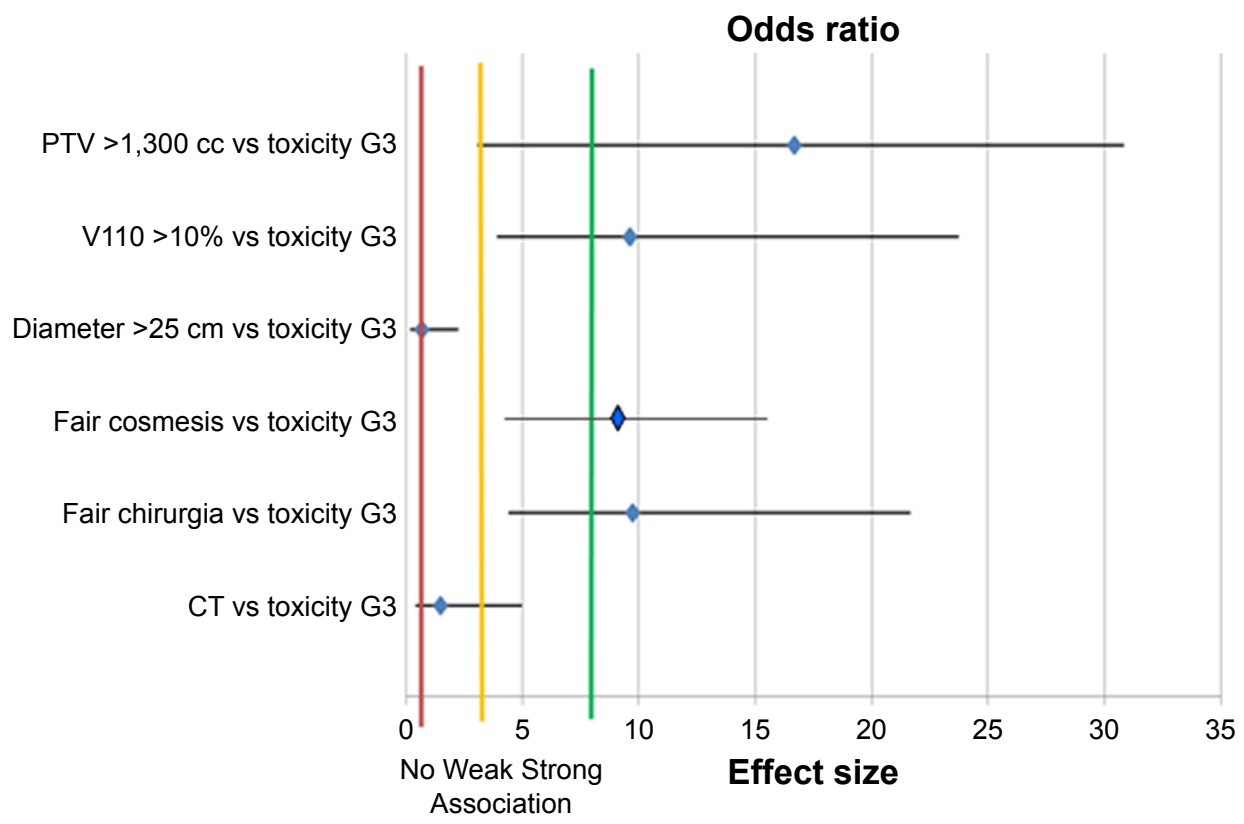

Figure 2 Forest plot for multivariate analysis.

Notes: Correlation between variables and toxicity: diamonds over the green line represent as strong correlation; diamond before the yellow line represents as weak correlation; and diamond on the red line represents no correlation.

Abbreviations: PTV, planning target volume; G, grade; VII0, volume of the PTV breast taking II0\% of the prescribed dose; CT, chemotherapy. 
and safe. ${ }^{6}$ But some criticisms have been moved because these results should have to be assessed also in a HF-WB 3D-CRT, which is nowadays a standard and adopted practice worldwide. ${ }^{15}$ According to a wide variety of hypofractionated schedules from randomized and not randomized trials including or not a boost, ${ }^{16}$ no homogeneous data regarding 3D dosimetry are available in the literature so the dosimetric parameters predictive for late toxicity and cosmetic outcome are still under investigation for each schedule. The presence of these biases has limited the widespread use of hypofractionation as a standard clinical practice so that the ASTRO consensus guidelines 2011 recommend hypofractionated whole-breast irradiation to patients older than 50 years, without chemotherapy and the maximum dose should not be $>107 \%$ of PD in the central axis plane encouraging the use of $3 \mathrm{D}$ planning to optimize treatment and resolve some dosimetric issues. ${ }^{9}$ In regard to the Ontario Canadian Trial, which was the treatment scheme of this retrospective analysis, all patients received a 2D radiotherapy technique, and only $10.9 \%$ of patients had adjuvant systemic therapy. The only dosimetric parameter to exclude patients from the trial was a BD $>25 \mathrm{~cm}$ at the posterior border of the medial and lateral tangential beams because it might influence dose inhomogeneity on a 2D radiation plan. Furthermore, in the results, the predictive factors of cosmetic outcome were age $<50$ years and time of randomization, which were the only features able to influence the results according to the follow-up time span. ${ }^{6}$

Radiobiological principles regulate hypofractionated radiotherapy that is delivered with larger daily fraction size in a shorter treatment time. ${ }^{17,18}$ It is widely accepted that the hotspots in large-sized fractions increase the dose/fraction and the total dose, leading to the so-called double-trouble and triple-trouble effects that could affect the tissues' tolerance and the therapeutic benefit. ${ }^{19,20}$ In fact, in a recent review, Yarnold et $\mathrm{al}^{8}$ questioned about the role of hotspots in hypofractionation and in the volume-receiving hotspots for the risk of late normal tissue complications. The linearquadratic model and NTCP proposed by Lyman are useful tools to calculate the biological equivalent dose, taking into account the larger dose/fraction and the risk of toxicity in the dose/volume ratio in $3 \mathrm{D}$ planning. ${ }^{21,22}$ Plotting the data of the total dose by hotspots of $10 \%$ and $20 \%$, a dose over $10 \%$ of the PD to more than $10 \%$ of the PTVbr (V110>10\%) was found to be a predictive parameter of breast damage, and it was confirmed by the G3 toxicity and worse cosmesis in the results. On the other hand, many clinical trials have been developed in Europe, Canada, and the USA assessing the effectiveness and safety of different fraction sizes and schedules with modern radiation techniques, so no homogeneous dosimetric constraints have been developed to fit every hypofractionation schedule and no consensus on hotspots/volume or breast size to be treated has been defined. For example, Ciammella et al, ${ }^{23}$ in a report on hypofractionated whole-breast radiotherapy (40.05 Gy in 15 daily fractions, $2.67 \mathrm{~Gy} /$ fraction with boost), identified V104 and V107 as factors related to chronic subcutaneous toxicity while the breast volume was related with fair-poor results. The impact of BD like a breast size surrogate is still controversial because many trials have excluded from hypofractionation large-breasted women while several other trials have treated these patients without providing clear information about their toxicity and cosmetic outcome. Moreover, there is not a standard criterion to define the optimal size of "large breast," which depends on multiple factors such as the position and inclination degree of the chest, the use of an immobilizing device, and the interobserver contouring operator variability. These biases may bring many discrepancies between toxicity and cosmetic outcomes among both obese, large-breasted and normal-breasted women recruited in the trials. ${ }^{24,25}$ Most recently, in a preliminary report by the Danish Breast Cancer Cooperative Group (DBCG) HYPO trial, the authors found the breast volume over $600 \mathrm{cc}$ as a predictive factor for breast induration. ${ }^{26}$ Tortorelli ${ }^{27}$ suggested that acute skin reaction was correlated with the amount of volume receiving $>107 \%$, but no information on late reaction is provided. In accordance to this report, Chen et $\mathrm{al}^{28}$ pointed out that the larger volume receiving $>53.9$ Gy has been a significant predictor of radiation-induced skin toxicity. Moreover, Goldsmith et $\mathrm{al}^{29}$ concluded that large-breasted women were more likely to suffer change in breast size and shape after hypofractionated whole-breast 3D radiotherapy, perhaps the dose inhomogeneity was not enough to explain this association. Deantonio et $\mathrm{al}^{30}$ on a hypofractionated wholebreast study (45 Gy in 20 fractions, 2.25 Gy/fr and 9 Gy boost) reported that breast volume increased the hazard of late toxicity over time (hazard ratio $=1.27,95 \% \mathrm{CI}=1.04-1.55$, and $P=0.016$ ) when analyzed with Cox's proportional hazards regression model. Despite these discrepancies, Tsang et al ${ }^{31}$ in a report on hypofractionated UK FAST trials showed that the dose heterogeneity does not impact on the risk of 2-year change photographic breast appearance after hypofractionation so in the START A and B Trial large breast volume is not considered an exclusion criterion for hypofractionation because the lower limit of the $\alpha / \beta$ ratio for adverse effects regarding breast appearance was $2.0 \mathrm{~Gy} .{ }^{31}$ Anyway, a modern 
technique such as intensity-modulated radiation therapy seems to solve overdoses in large breasts as reported by Hannan et al. ${ }^{32}$ In our study, there was no significant correlation between BD (as surrogate of dose heterogeneity) with late toxicity and cosmesis although a PTVbr over 1,300 cc significantly correlated with greater toxicity as seen in the univariate and multivariate analyses. In regard to the role of adjuvant chemotherapy in breast cancer, the DBCG-82TM protocol $^{33}$ and a multivariate analysis by $\mathrm{Recht}^{34}$ have reported a negative impact of chemotherapy on cosmetic outcome and late normal tissue reactions after standard whole-breast radiotherapy but no many data are available with hypofractionation trials. In fact, $\sim 1,600$ patients have received adjuvant chemotherapy and only $10.9 \%$ of the recruited patients in the Ontario Canadian Trial. Moreover, the subgroup analysis of this trial has shown similar toxicity regardless of estrogen receptors and chemotherapy. ${ }^{6}$ Anyway, most recently, Hijal et $\mathrm{al}^{35}$ observed no late adverse effects of chemotherapy in combination with hypofractionated wholebreast radiotherapy as we found in our analysis.

At least in the Ontario Canadian Trial, significant predictive factors of negative cosmetic outcome were age $<50$ years and time from randomization $(P<0.001)$. In our clinical practice, postmenopausal women over 60 years were recruited to avoid age-related bias.

Another question is the time span of follow-up to validate the role of the reported results. In fact, there are some critical questions regarding the impact of the entire life span of follow-up on normal tissue toxicity and cosmetic outcome in hypofractionation at the time of reporting data, because late-responding tissues need more time to develop definitive effects.

Curran et $\mathrm{al}^{36}$ reported that cosmesis after breast-conserving therapy worsened in patients with a follow-up longer than 5 years. Ciammella et $\mathrm{al}^{23}$ reported an overall low risk of late skin toxicity in patients with a median follow-up of 34 months. In our study, the data are lasting from a median follow-up of 60 months and probably a longer follow-up time could modify the results. Anyway, other authors did not show any difference between 5-year and 10-year late adverse effects. ${ }^{15}$ On the other hand, in the Ontario Canadian Trial, data at 5 years of follow-up showed in the experimental arm a 79\% for the excellent-good cosmetic outcome and a G2-G3 skin and subcutaneous morbidity score less than $4 \%$. In our experience at a median follow-up of 60 months, results of good cosmesis have been recorded in $80 \%$ of patients while G3 skin toxicity has been nearly $5 \%$ in comparison to $4 \%$ of the Canadian experience.

\section{Conclusion}

Hypofractionated radiotherapy is more convenient and preferred by patients and physicians and has been associated with more prompt recovery and improved quality of life than longer radiotherapy courses. ${ }^{3}$ In answer to some reasonable criticisms moved before, ${ }^{8}$ the results of this retrospective study using the Canadian hypofractionated whole-breast adjuvant conformal $3 \mathrm{D}$ radiotherapy in our clinical practice for postmenopausal early breast cancer patients pointed out the role of dosimetric parameters rather than clinical factors in probability of breast complications and worse cosmetic outcomes. In fact, the NTCP model has been a useful tool able to find a dosimetric parameter (V110 <10\% of PTVbr), which predicts good cosmesis and safety on normal tissue reaction. Thus, it can be used to select patients eligible for hypofractionation. The issue of breast size being measured in volume over $1,300 \mathrm{cc}$ rather than the $25 \mathrm{~cm}$ cut-off for BD has been an adjunctive factor impacting on cosmesis and breast late damage, showing an important predictive role. The evaluation of these parameters in routine clinical practice could allow the establishment of a gentler and safe hypofractionated regimen. A longer follow-up time is needed to validate the results.

\section{Acknowledgment}

The authors thank MP Ciliberti MD, G Laterza PhD, R Marchese MD, AR Marsella MD, MG Monis MD, G Porrazzo MD, and M Soloperto MD for their contribution.

\section{Disclosure}

The authors report no conflicts of interest in this work.

\section{References}

1. Koulis TA, Phan T, Olivotto IA. Hypofractionated whole breast radiotherapy: current perspectives. Breast Cancer. 2015;7:363-370.

2. Versmessen H, Vinh-Hung V, Van Parijs H, et al. Health-related quality of life in survivors of stage I-II breast cancer: randomized trial of postoperative conventional radiotherapy and hypofractionated tomotherapy. BMC Cancer. 2012;12:495.

3. Zhou ZR, Mei X, Chen XX, et al. Systematic review and meta-analysis comparing hypofractionated with conventional fraction radiotherapy in treatment of early breast cancer. Surg Oncol. 2015;(24)3:200-211.

4. Budach W, Bolke E, Matuschek C. Hypofractionated radiotherapy as adjuvant treatment in early breast cancer. A review and meta-analysis of randomized controlled trials. Breast Care. 2015;10:240-245.

5. Whelan T, MacKenzie R, Julian J, et al. Randomized trial of breast irradiation schedules after lumpectomy for women with lymph nodenegative breast cancer. J Natl Cancer Inst. 2002;94:1143-1150.

6. Whelan TJ, Pignol JP, Levine MN, et al. Long-term results of hypofractionated radiation therapy for breast cancer. $N$ Engl J Med. 2010;362: 513-520.

7. Williams MV, Denekemp J, Fowler JF. A review of alpha/beta ratios for experimental tumors: implications for clinical studies of altered fractionation. Int J Radiat Oncol Biol Phys. 1985;11:87-96. 
8. Yarnold J, Bentzen SM, Coles C, Haviland J. Hypofractionated wholebreast radiotherapy for women with early breast cancer: myths and realities. Int J Radiat Oncol Biol Phys. 2011;79:1-9.

9. Smith BD, Bentzen SM, Correa CR, et al. Fractionation for whole breast irradiation: an American Society for Radiation Oncology (ASTRO) evidence-based guideline. Int J Radiat Oncol Biol Phys. 2011;81(1):59-68.

10. Silvano G, Lazzari G, Marsella AR, et al. Postoperative Hypofractionated Whole Breast Radiotherapy in patients with early stage breast cancer: interim cosmetic and toxicity evaluation. Int J Radiat Oncol Biol Phys. 2008;72(1 Suppl):S193.

11. Aaronson NK, Bartelink H, van Dongen JA, van Dam FS. Evaluation of breast conserving therapy: clinical, methodological and psycosocial perspectives. Eur J Surg Oncol. 1988;14:133-140.

12. Cox JD, Stetz J, PajaK TF. Toxicity Criteria of the Radiation Therapy Oncology Group (RTOG) and the European Organization for Research and Treatment of Cancer (EORTC). Int J Radiat Oncol Biol Phys. 1995;31:1341-1346.

13. Taylor ME, Perez CA, Halverson KJ, et al. Factors influencing cosmetic results after conservation therapy for breast cancer. Int J Radiat Oncol Biol Phys. 1995;31(4):753-764.

14. Kacprowska A, Jassem J. Hypofractionated radiotherapy for early breast cancer: review of phase III studies. Rep Pract Oncol Radiother. 2012;17:66-70.

15. Yarnold J, Ashton A, Bliss J, et al. Fractionation sensitivity and dose response of late adverse effects in the breast after radiotherapy for early breast cancer: long-term results of a randomized trial. Radiother Oncol. 2005;75:9-17.

16. Haviland JS, Owen JR, Dewar JA, et al; START Trialists' Group. The UK Standardisation of Breast Radiotherapy (START) trials of radiotherapy hypofractionation for treatment of early breast cancer: 10-year follow-up results of two randomised controlled trials. Lancet Oncol. 2013;14:1086-1094.

17. Owen JR, Ashton A, Bliss JM, et al. Effects of radiotherapy fraction size on tumour control in patients with early-stage breast cancer after local tumour excision: long-term results of a randomized trial. Lancet Oncol. 2006;7:467-471.

18. Lyman JT. Complication probability as assessed from dose-volume histograms. Radiat Res Suppl. 1985;8:S13-S19.

19. Bentzen SM, Yarnold JR. Reports of unexpected late side-effects of accelerated partial breast irradiation - radiobiological considerations. Int J Radiat Oncol Biol Phys. 2010;77(4):969-973.

20. Dale RG. The application of the linear-quadratic dose-effect equation to fractionated and protracted radiotherapy. Br J Radiol. 1985;58: $515-528$.

21. Fowler JF. The linear-quadratic formula and progress in fractionated radiotherapy. Br J Radiol. 1989;62:679-694.

22. Jones B, Dale RG, Deehan C, Hopkins KI, Morgan DA. The role of biologically effective dose (BED) in clinical oncology. Clin Oncol (R Coll Radiol). 2001;13:71-81.
23. Ciammella P, Podgornii A, Galeandro M, et al. Toxicity and cosmetic outcome of hypofractionated whole-breast radiotherapy: predictive clinical and dosimetric factors. Radiat Oncol. 2014;9:97.

24. Plataniotis GA, Dale RG. Biologically effective dose-response relationship for breast cancer treated by conservative surgery and postoperative radiotherapy. Int J Radiat Oncol Biol Phys. 2009;75:512-517.

25. Corbin KS, Dorn PL, Jain SK, Al-Hallaq HA, Hasan Y, Chmura SJ. Hypofractionated radiotherapy does not increase acute toxicity in large-breasted women: results from a prospectively collected series. Am J Clin Oncol. 2014;37(4):322-326.

26. Offersen BV, Nielsen HM, Jacobsen EH, et al. Hypo- vs normofractionated radiation of early breast cancer in the randomized DBCG HYPO trial. Radiother Oncol. 2016;119(Suppl 1):S64-S65.

27. Tortorelli G, Di Murro L, Barbarino R, et al. Standard or hypofractionated radiotherapy in the postoperative treatment of breast cancer: a retrospective analysis of acute skin toxicity and dose inhomogeneities. BMC Cancer. 2013;13:230.

28. Chen MF, Chen WC, Lai CH, Hung CH, Liu KC, Cheng YH. Predictive factors of radiation-induced skin toxicity in breast cancer patients. BMC Cancer. 2010;10:508.

29. Goldsmith C, Haviland J, Tsang Y, Sydenham M, Yarnold J; FAST Trialists' Group. Large breast size as a risk factor for late adverse effects of breast radiotherapy: is residual dose inhomogeneity, despite 3D treatment planning and delivery, the main explanation? Radiother Oncol. 2011;100(2):236-240.

30. Deantonio L, Gambaro G, Beldì D, et al. Hypofractionated radiotherapy after conservative surgery for breast cancer: analysis of acute and late toxicity. Radiat Oncol. 2010;5:112.

31. Tsang Y, Haviland J, Venables K, Yarnold J; FAST Trial Management Group. The impact of dose heterogeneity on late normal tissue complication risk after hypofractionated whole breast radiotherapy. Radiother Oncol. 2012;104(2):143-147.

32. Hannan R, Thompson RF, Chen Y, et al. Hypofractionated whole-breast radiation therapy: does breast size matter? Int J Radiat Oncol Biol Phys. 2012;84(4):894-901.

33. Johansen J, Overgaard J, Overgaard M. Effect of adjuvant systemic treatment on cosmetic outcome and late normal-tissue reactions after breast conservation. Acta Oncol. 2007;46:525-533.

34. Recht A. Integration of systemic therapy and radiation therapy for patients with early-stage breast cancer treated with conservative surgery. Clin Breast Cancer. 2003;4:104-113.

35. Hijal T, Al Hamad AA, Niazi T, et al. Hypofractionated radiotherapy and adjuvant chemotherapy do not increase radiation-induced dermatitis in breast cancer patients. Curr Oncol. 2010;17(5):22-27.

36. Curran D, van Dongen JP, Aaronson NK, et al. Quality of life of earlystage breast cancer patients treated with radical mastectomy or breastconserving procedures: results of EORTC Trial 10801. Eur J Cancer. 1998;34:307-314
OncoTargets and Therapy

\section{Publish your work in this journal}

OncoTargets and Therapy is an international, peer-reviewed, open access journal focusing on the pathological basis of all cancers, potential targets for therapy and treatment protocols employed to improve the management of cancer patients. The journal also focuses on the impact of management programs and new therapeutic agents and protocols on
Dovepress

patient perspectives such as quality of life, adherence and satisfaction. The manuscript management system is completely online and includes a very quick and fair peer-review system, which is all easy to use. Visit http://www.dovepress.com/testimonials.php to read real quotes from published authors. 